\title{
Binary solvent mixtures: characterization of molecular environments through multiparametric empirical scales. Concerns about relationships between kinetic data and microscopic solvent properties
}

\author{
Pedro M. Mancini*, Alexis Bock, Claudia Adam, Adriana del C. Pérez, and Leonor R. \\ Vottero
}

Departamento de Química, Área Química Orgánica, Facultad de Ingeniería Química, Universidad Nacional del Litoral, Santiago del Estero 2829, (3000) Santa Fe, Argentina E-mail:pmancini@fiqus.unl.edu.ar

Dedicated with appreciation to Professor Edmundo Rúveda and to Professor Roberto Rossi (received 17 Jul 03; accepted 08 Oct 03; published on the web 10 Oct 03)

\begin{abstract}
The microscopic solvent properties dipolarity/polarizability SPP, basicity SB and acidity SA were determined for binary mixtures of ethyl acetate with chloroform or acetonitrile or methanol as cosolvent. Each solvent system was analyzed according to its deviation from ideal behavior due to the preferential solvation phenomenon. The results were related to the dipolarity/polarizability $\pi^{*}$, basicity $\beta$ and acidity $\alpha$ evaluating, in each case, the concordance between both multiparametric scales. The solvent properties were correlated with the kinetic properties corresponding to a nucleophilic aromatic substitution reaction through linear free energy relationships. The adequacy of the multiparametric scales as appropriate approaches to describe the solvent features in microscopic environments was analyzed.
\end{abstract}

Keywords: Multiparametric solvent scales, dipolarity/polarizability, basicity, acidity, concordance, correlations

\section{Introduction}

Previous studies have been reported by us ${ }^{1}$ which have contributed to the prediction and description of solvent effects on solvatochromic and kinetic processes. On the one hand, we characterized several binary solvent mixtures by determining the solvatochromic solvent parameters $E_{\mathrm{T}}(30)$ (Dimroth-Reichardt) ${ }^{2}$ and $\pi^{*}, \alpha$ and $\beta$ (Kamlet, Abboud and Taft - KAT-) ${ }^{3}$ which describe empirically the solvation interactions at a molecular level. On the other hand, we explored the kinetics of some nucleophilic aromatic substitution reactions performed in mixed 
solvents. In each case, the intermolecular interactions taking place and the preferential solvation phenomenon were analyzed. The solvatochromic and the kinetic solvent-dependent processes have been related. In general, the mixed solvents explored were those in which the components are solvent-active to one another forming associated species through specific molecular interactions. ${ }^{4,5}$

In order to contribute to the rationalization of the treatment of the solvent effects, the purpose of this work is to extend the precedent analysis to the solvent dipolarity/polarizability SPP, solvent acidity SA and solvent basicity SB (scales reported by Catalán and co-workers) which have not yet been used extensively. ${ }^{6}$ The general purpose of this work, though, is to evaluate if empirical solvent parameters provide an appropriated framework to the interpretation of medium effects on different processes occurring in mixed solvents.

\section{Results and Discussion}

Based on the application of Kamlet and Taft's solvatochromic comparison method (SCM), the SPP scale was drawn from the solvatochromic shifts undergone by the longest wavelength absorption maximum of two indicators: 2-(dimethylamino)-7-nitrofluorene (DMANF) and 2fluoro-7-nitrofluorene (FNF). The SB and SA scales are based on the probe-homomorphs couples 5-nitroindoline (NI) and 1-methyl-5-nitroindoline (MNI), and o-tert-butylstilbazolium (TBSB) and $o, o^{\prime}$-di-tert-butylstilbazolium (DTBSB) betaine dyes, respectively. The parameters were calculated from the experimental wavenumbers of the absorption maximum of the solvatochromic solutes according to the equations1-3. ${ }^{6 \mathrm{a}, \mathrm{c}, \mathrm{e}}$ The deconvolution of the experimental spectra was applied for the calculation of SA values. ${ }^{6}$

$$
\begin{array}{ll}
\mathrm{SPP}=\Delta \tilde{v} \text { (solvent)- 4692/2119 } & \Delta \tilde{v}\left(\mathrm{~cm}^{-1}\right)=\tilde{v}_{\mathrm{FNF}}-\tilde{v}_{\mathrm{DMANF}} \\
\mathrm{SB}=(\Delta \tilde{v}-1570) /-1735 & \Delta \tilde{v}\left(\mathrm{~cm}^{-1}\right)=\tilde{v}_{\mathrm{NI}}-\tilde{v}_{\mathrm{MNI}} \\
\mathrm{SA}=(\Delta \tilde{v} / 1299.8) 0.4 & \Delta \tilde{v}\left(\mathrm{~cm}^{-1}\right)=\tilde{v}_{\mathrm{TBSB}}-\left(1.4099 \tilde{v}_{\mathrm{DTBS}}-6288.7\right)
\end{array}
$$

Recently, some solvent mixtures were characterized following the pure solvent scales mentioned above. ${ }^{6 \mathrm{~g}, \mathrm{~h}}$ Moreover, the SPP scale has been analyzed in terms of polarity and polarizability contributions. It might be possible that polarity predominates. ${ }^{7}$

In this work, binary mixtures of ethyl acetate ( $\mathrm{EAc}, \mathrm{SPP}=0.795, \mathrm{SB}=0542, \mathrm{SA}=0)^{6}$ with chloroform ( $\mathrm{SPP}=0.786, \mathrm{SB}=0.071, \mathrm{SA}=0.047)^{6}$ or acetonitrile $(\mathrm{AcN}, \mathrm{SPP}=0.895, \mathrm{SB}=0.286$, $\mathrm{SA}=0.044)^{6}$ or methanol $(\mathrm{MeOH}, \mathrm{SPP}=0.857, \mathrm{SB}=0.545, \mathrm{SA}=0.605)^{6}$ were characterized. In these solvent systems, specific intersolvent interactions by hydrogen-bonding are involved. ${ }^{2,3}$ These selected mixtures are part of the set of binary mixtures previously characterized by $\pi^{*}, \alpha$ and $\beta$ parameters. ${ }^{1 \mathrm{ab}, \mathrm{c}}$ 
The solvatochromic parameters SPP, SB and SA, which were calculated from the experimental wavenumbers determined by us for the proposed mixtures, are presented in Table 1. Figure 1 shows the variation of the properties on the overall cosolvent molar fraction range.

Table 1. SPP, SB and SA solvent properties for (Ethyl Acetate + cosolvent) binary mixtures

\begin{tabular}{|c|c|c|c|c|c|c|c|c|c|}
\hline & \multicolumn{9}{|c|}{ Cosolvent molar fraction } \\
\hline & 0.1 & 0.2 & 0.3 & 0.4 & 0.5 & 0.6 & 0.7 & 0.8 & 0.9 \\
\hline & \multicolumn{9}{|c|}{ Chloroform } \\
\hline SPP & 0.713 & 0.726 & 0.790 & 0.781 & 0.860 & 0.858 & 0.825 & 0.778 & 0.76 \\
\hline SB & 0.549 & 0.573 & 0.583 & 0.588 & 0.553 & 0.505 & 0.456 & 0.381 & 0.215 \\
\hline SA & 0.011 & 0.021 & 0.029 & 0.050 & 0.057 & 0.044 & 0.029 & 0.029 & 0.027 \\
\hline
\end{tabular}

Acetonitrile

$\begin{array}{lccccccccc}\text { SPP } & 0.800 & 0.830 & 0.776 & 0.860 & 0.862 & 0.915 & 0.913 & 0.867 & 0.878 \\ \text { SB } & 0.545 & 0.558 & 0.567 & 0.557 & 0.494 & 0.441 & 0.416 & 0.406 & 0.363 \\ \text { SA } & 0 & 0 & 0 & 0 & 0 & 0 & 0.004 & 0.044 & 0.044\end{array}$

Methanol

\begin{tabular}{llllllllll} 
SPP & 0.752 & 0.781 & 0.803 & 0.788 & 0.787 & 0.802 & 0.818 & 0.822 & 0.820 \\
SB & 0.546 & 0.592 & 0.633 & 0.619 & 0.600 & 0.598 & 0.581 & 0.560 & 0.547 \\
SA & 0.035 & 0.238 & 0.243 & 0.262 & 0.300 & 0.334 & 0.400 & 0.482 & 0.530 \\
\hline
\end{tabular}
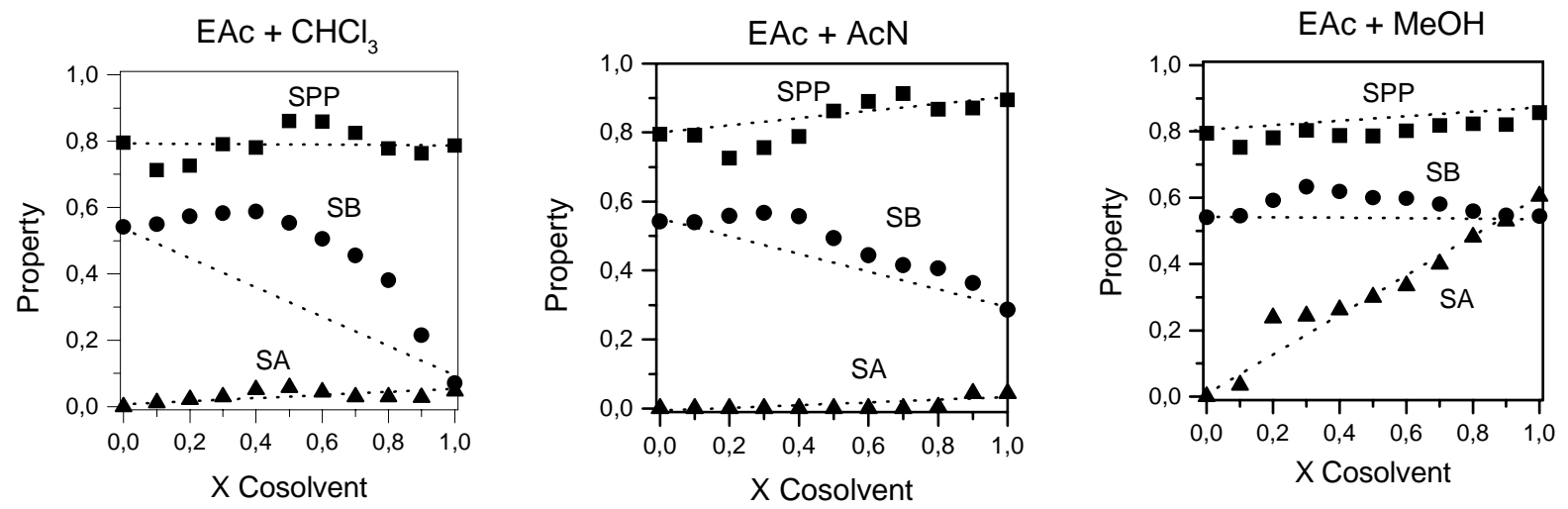

Figure 1. Plots of SPP, SB and SA parameters vs cosolvent molar fraction. 
The SPP values vary in a special way according to those previously reported ${ }^{6 a, b, g, h}$ for the cases in which the pure components of the mixtures have almost the same polarity/polarizability. The SA values corresponding to the mixtures of (EAc $+\mathrm{CHCl}_{3}$ or $\mathrm{AcN}$ ) exhibit negligible acidity throughout the cosolvent molar fraction range. The system (EAc $+\mathrm{MeOH}$ ) (in which the acidity of the pure components differs in 0.6 units) shows high increases in the property values with the addition of $\mathrm{MeOH}$ to pure EAc, reaching the ideal behavior in the $\mathrm{MeOH}$-rich zone.

The SB values exhibit positive deviations from the ideal behavior at the whole cosolvent molar fractions range. Moreover, synergetic effect on the property is observed for mixtures with cosolvent molar fractions lower than 0,50 in the case of $\mathrm{CHCl}_{3}, 0,40$ in the case of $\mathrm{AcN}$ and at all molar fractions in the case of $\mathrm{MeOH}$. It is possible to observe that by enriching the mixtures with the cosolvent, the basicity decreases rapidly above $\mathrm{X}_{\mathrm{CoS}}=0.5$.

\section{Comparison of SPP, SA and SB with $\pi^{*}, \alpha$ and $\beta$ : evaluation of the concordance between both multiparametric empirical scales}

The degree of agreement between the values for each property measured by different scales was evaluated through the Bland-Altman (B-A) approach. ${ }^{8}$ This is a statistical technique helpful in analyzing whether two different methods of measurement agree sufficiently closely. The B-A analysis includes: i) the calculation of the difference of each pair of measurements; ii) the calculation of the mean of the differences; iii) the determination of the standard deviation of the differences; iv) the determination of the 95\% confidence intervals on the differences; and iv) to contruct a plot of the differences against the means indicating with horizontal lines the mean differences and the 95\% confidence intervals (concordance limits). The results are shown in Table 2. The B-A plots are shown in Figures 2.

Table 2. Mean of the differences (DM), standard deviation (SD) and 95\% confidence intervals (CI). Student's test value $(t)=2,228$ for 10 degrees of freedom. (a) ${ }^{1 \mathrm{a}, \mathrm{b}, \mathrm{c}}$

\begin{tabular}{ccccc}
\hline \multirow{2}{*}{ Cosolvent } & Parameters & DM & SD & $95 \%$ CI \\
& & & & \\
\hline $\mathrm{CHCl}_{3}$ & SPP vs $\pi^{*^{\mathrm{a}}}$ & 0,177 & 0,066 & $0,323 / 0,031$ \\
& SB vs $\beta^{\text {a }}$ & 0,076 & 0,089 & $0,274 /-0,122$ \\
& SA vs $\alpha^{\mathrm{a}}$ & $-0,117$ & 0,062 & $0,021 /-0,255$ \\
$\mathrm{AcN}$ & SPP vs $\pi^{*^{\mathrm{a}}}$ & 0,171 & 0,045 & $0,272 / 0,071$ \\
& SB vs $\beta^{\text {a }}$ & 0,002 & 0,085 & $0,191 /-0,188$ \\
& SA vs $\alpha^{\mathrm{a}}$ & $-0,222$ & 0,101 & $0,004 /-0,447$ \\
$\mathrm{MeOH}$ & SPP vs $\pi^{*}$ & 0,214 & 0,024 & $0,267 / 0,161$ \\
& SB vs $\beta^{\mathrm{a}}$ & $-0,027$ & 0,061 & $0,108 /-0,162$ \\
& SA vs $\alpha^{\mathrm{a}}$ & $-0,479$ & 0,173 & $-0,094 /-0864$ \\
\hline
\end{tabular}


$\mathrm{EAc}+\mathrm{CHCl}_{3}$
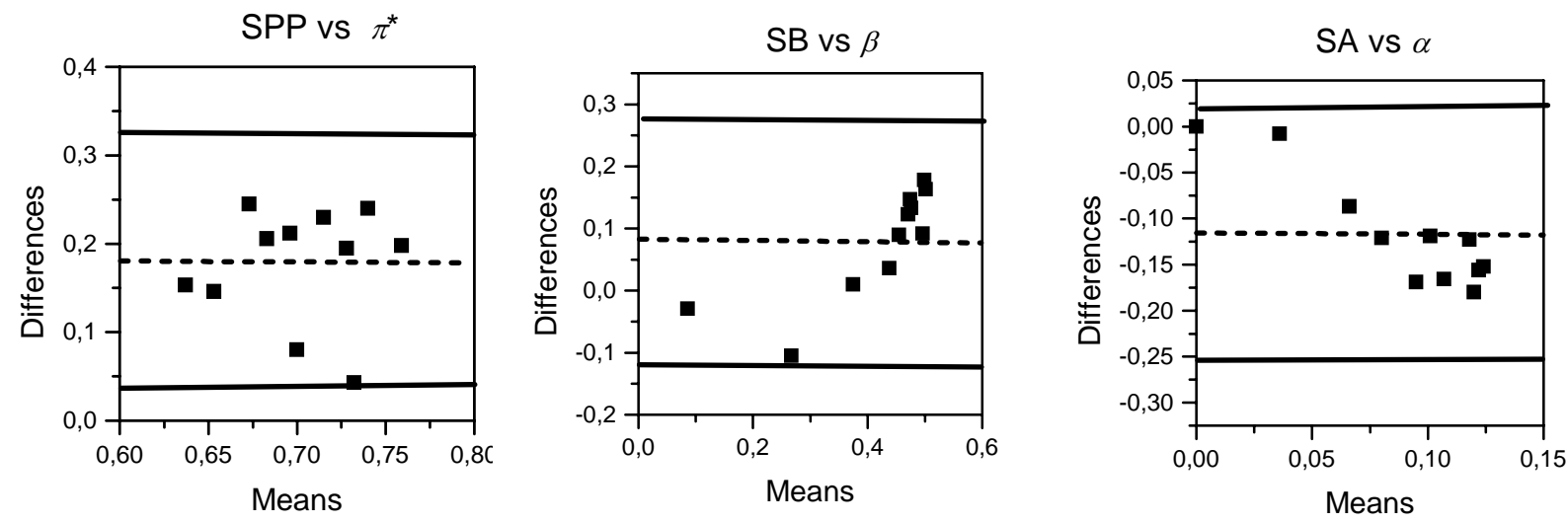

$\mathrm{EAc}+\mathrm{AcN}$
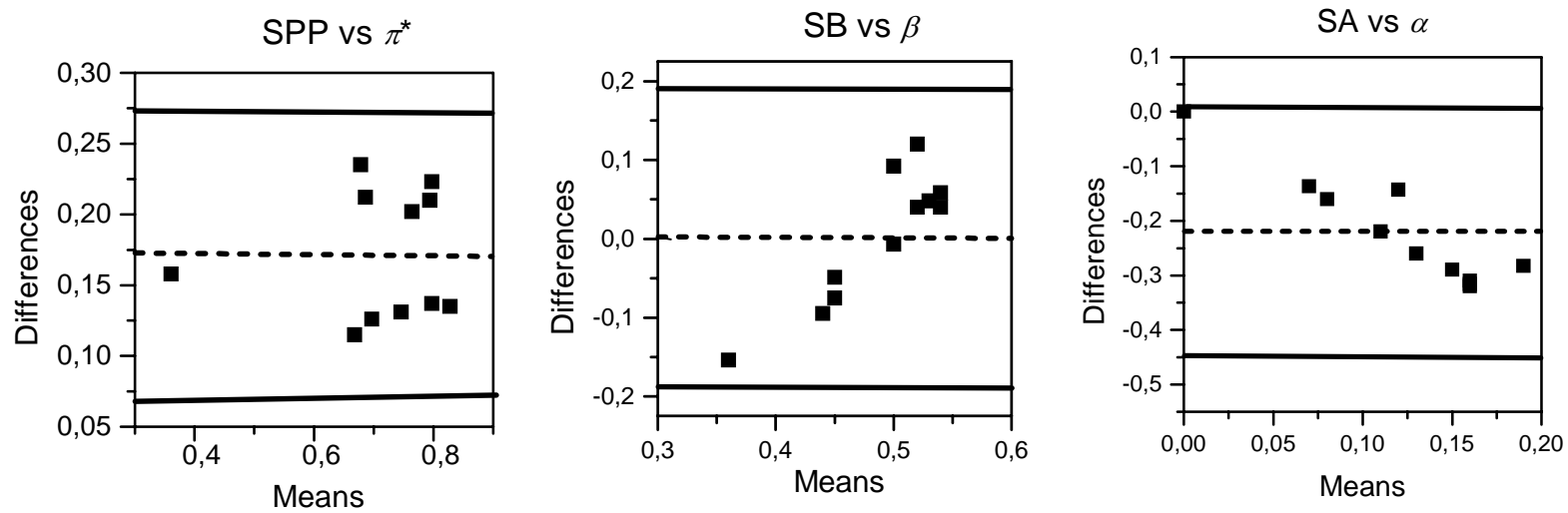

\section{$\mathrm{EAc}+\mathrm{MeOH}$}
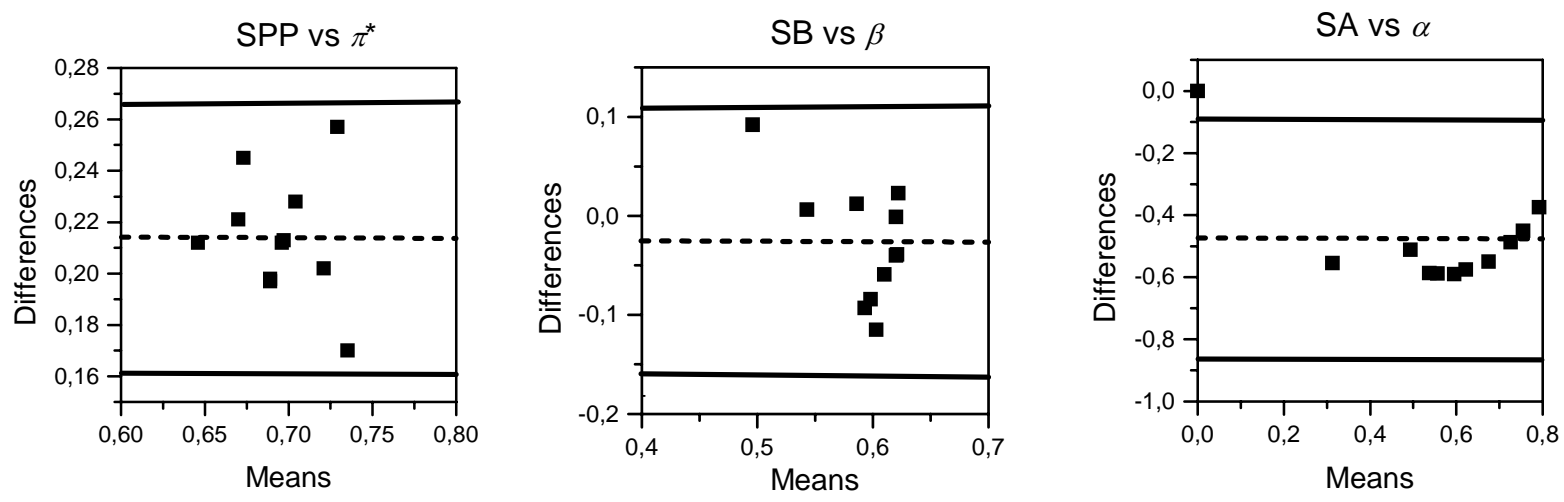

Figure 2. Plots of Difference between parameters vs Mean of the parameters. Mean differences (----). Limits of concordance (-). 
As can be observed in the B-A plots, the difference values do not exceed the concordance limit, these being distributed randomly from one side to the other of the line which corresponds to difference zero. In most cases, the differences do not vary in any systematic way over the range of measurements.

\section{Correlation between kinetic properties and microscopic solvent properties}

As a contribution to the discussion whether solvatochromic scales are adequate tools to describe solvent effects on any chemical process, Linear Free Energy Relationships ${ }^{9}$ (LFERs) between logarithm of rate constants and empirical solvent parameters were investigated. Moreover, the agreement of different but comparable three-parametric models to interpret the solvation effects on a kinetic process was discussed.

On the one hand, the kinetic study of the aromatic nucleophilic substitution $\left(\mathrm{S}_{\mathrm{N}} \mathrm{Ar}\right)$ reaction between 1-fluoro-2,4-dinitrobenzene (FDNB) and the secondary amine morpholine (Mo) in EAc $+\mathrm{CHCl}_{3}$ or $\mathrm{MeOH}$ mixtures has been previously reported. ${ }^{1 \mathrm{a}, \mathrm{c}}$ On the other hand, the $\pi^{*}, \alpha$ and $\beta$ microscopic parameters corresponding to these binary mixtures have been previously determined. ${ }^{1 \text {,d,e }}$ The correlation analysis between the second-order rate constant $\left(k_{\mathrm{A}}\right)$ (at low and high Mo concentration) and SPP, SB and SA, as $\pi^{*}, \alpha$ and $\beta$ solvent descriptors, are shown in Table 3.

The correlations are acceptable for both multiparametric approaches. The results clearly show that Catalán's equation exhibit better correlations than KAT's equation when the cosolvent is $\mathrm{MeOH}$, while opposite results are observed when the cosolvent is $\mathrm{CHCl}_{3}$.

From the signs and values of the $s, a$ and $b$ correlation coefficients, mostly analogies can be observed between both multiparametric approachs, but there are some differences as well:

- The influence of the solvation effects attributed to the dipolarity/polarizability of the solvent on the reaction rate is negative except from the $\mathrm{MeOH}$ mixtures at high Mo concentration (in which it is positive according to the Catalán approach) and $\mathrm{CHCl}_{3}$ mixtures at low Mo concentration (in which it is not significant according to KAT approach).

- The impact of the hydrogen bond acceptor character of the solvent on the reaction rate is positive in all cases except from the $\mathrm{MeOH}$ mixtures at high Mo concentration (in which it is negative according to both multiparametric approaches).

- The influence of the hydrogen bond donor ability of the solvent on the reaction rate is positive at low Mo concentration and negative at high Mo concentration except from $\mathrm{CHCl}_{3}$ mixtures at high Mo concentration (in which it is not significant according to KAT approach) 
Table 3. Correlation data for the reaction of 2,4-DNFB with Mo in (EAc $\left.+\mathrm{CHCl}_{3}\right)$ and (EAc + $\mathrm{MeOH})$ mixtures: correlation coefficient $\left(r\right.$ and $r^{2}$ ), standard deviation (SD), intercept $(Y)$ and parameters $s, a$ and $b$ (and their standard errors)

\begin{tabular}{|c|c|c|c|c|c|}
\hline \multicolumn{2}{|c|}{ [amine] } & \multicolumn{2}{|c|}{$\log k_{\mathrm{A}}=Y+s \mathrm{SPP}+b \mathrm{SB}+a \mathrm{SA}$} & \multicolumn{2}{|c|}{$\log k_{\mathrm{A}}=Y+s \pi^{*}+b \beta+a a$} \\
\hline \multirow{7}{*}{ [0.002] } & & & & & \\
\hline & $r\left(r^{2}\right)$ & 0.957 & $(0.916)$ & 0.987 & $(0.975)$ \\
\hline & $S D$ & 0.078 & & 0.043 & \\
\hline & $Y\left(s_{Y}\right)$ & -0.246 & $(0.455)$ & -2.009 & $(0.205)$ \\
\hline & $s\left(s_{s}\right)$ & -1.755 & $(0.662)$ & 0.339 & $(0.292)$ \\
\hline & $b\left(s_{b}\right)$ & 1.476 & $(0.174)$ & 1.907 & $(0.144)$ \\
\hline & $a\left(s_{a}\right)$ & 7.667 & (1.979) & 2.385 & $(0.208)$ \\
\hline \multirow[t]{6}{*}{ [0.080] } & $r\left(r^{2}\right)$ & 0.921 & (0.849) & 0.964 & $(0.929)$ \\
\hline & $S D$ & 0.195 & & 0.133 & \\
\hline & $Y\left(s_{Y}\right)$ & 0.391 & (1.135) & 1.713 & $(0.634)$ \\
\hline & $s\left(s_{s}\right)$ & -1.786 & (1.650) & -4.973 & $(0.903)$ \\
\hline & $b\left(s_{b}\right)$ & 2.235 & $(0.434)$ & 3.171 & $(0.444)$ \\
\hline & $a\left(s_{a}\right)$ & -4.315 & (4.935) & 0.042 & $(0.642)$ \\
\hline \multicolumn{6}{|c|}{$\mathrm{MeOH}$} \\
\hline \multirow[t]{6}{*}{ [0.002] } & $r\left(r^{2}\right)$ & 0.990 & $(0.979)$ & 0.942 & $(0.887)$ \\
\hline & $S D$ & 0.047 & & 0.108 & \\
\hline & $Y\left(s_{Y}\right)$ & 0.496 & $(0.905)$ & -2.249 & (1.303) \\
\hline & $s\left(s_{s}\right)$ & -5.001 & $(1.037)$ & -0.553 & $(1.631)$ \\
\hline & $b\left(s_{b}\right)$ & 4.644 & $(0.466)$ & 3.542 & (2.978) \\
\hline & $a\left(s_{a}\right)$ & 1.703 & $(0.146)$ & 0.165 & (0.610) \\
\hline \multirow[t]{6}{*}{ [0.080] } & $r\left(r^{2}\right)$ & 0.974 & $(0.948)$ & 0.949 & $(0.901)$ \\
\hline & $S D$ & 0.045 & & 0.063 & \\
\hline & $Y\left(s_{Y}\right)$ & -1.709 & $(0.880)$ & 1.690 & $(0.756)$ \\
\hline & $s\left(s_{s}\right)$ & 2.616 & (1.008) & -1.542 & $(0.946)$ \\
\hline & $b\left(s_{b}\right)$ & -0.008 & $(0.454)$ & -0.972 & (1.728) \\
\hline & $a\left(s_{a}\right)$ & -1.152 & $(0.142)$ & -0.213 & $(0.354)$ \\
\hline
\end{tabular}

Number of data points $=11$ ( 9 binary mixtures +2 pure solvents $)$. 


\section{Conclusions}

The obtained results lead us to conclude that, for the explored solvent systems:

- In general, concordance is observed between different parameter scales which measure the same solvent property.

- In a first instance, both LSERs approaches are appropriate methods for quantification of the main interactions which affect the explored reaction.

\section{Experimental Section}

General Procedures. The solvatochromic indicators were prepared and/or purified as described elswhere. ${ }^{6}$ The solvents used were purified as previously reported and were kept over molecular sieves. ${ }^{1}$ The binary mixtures and the indicators solutions were prepared prior to use.

The spectroscopic data were obtained with a Perkin Elmer model Lambda $40 \mathrm{UV}$-Vis spectrophotometer equipped with a thermostatic cell holder. For each system explored, the property values were systematically determined throughout the total solvent composition range (at nine mixed solvent compositions) at $25^{\circ} \mathrm{C}$. The data treatment was carried out using the SSPS 10.0 Program and the SYSTAT PeakFit v4.11 Program.

\section{Acknowledgments}

This work received financial support from the Universidad Nacional del Litoral (Santa Fe, Argentina) through the Science and Technology Secretariat, UNL, CAI+D Program (Projects: 2000-17-151, 2002-21-152 and 2002-21-153).

\section{References}

1. (a) Mancini, P. M.; Terenzani, A.; Adam C.; Pérez A.; Vottero L. R. J. Phys. Org. Chem. 1999, 12, 207. (b) ibid. 713. (c) Mancini, P. M.; Adam, C.; Pérez A.; Vottero L. R. J. Phys. Org. Chem. 2000, 13, 221. (d) Mancini, P. M.; Pérez, del C.; Vottero L. R. J. Sol. Chem. 2001, 30, 695. (e) Mancini, P. M.; Pérez, del C.; Vottero L. R. Phys. Chem. Liq. 2003, 41, 45.

2. Reichardt, C. Solvent and Solvent Effects in Organic Chemistry Verlag Chemie: Weinheim, $19882^{\text {nd }}$ Edn.

3. (a) Abboud J-L. M., Kamlet M. J.; Taft R. W. Prog. Phys. Org. Chem. 1981, 13, 485. (b) Abboud J-L. M.; Notario R. Pure Appl. Chem. 1999, 71, 645.

4. (a) Dohnal V.; Costas M. J. Sol. Chem. 1996, 25, 635. (b) Tkadlecová, M.; Dohnal V.; Costas M. Phys. Chem. Chem. Phys. 1999, 1, 1479. 
5. Reimers J. R.; Hall L. E. J. Am. Chem Soc. 1999, 121, 3730.

6. (a) Catalán J., López V., Pérez P. Martín-Villamil R.; Rodríguez J.-G. Liebigs Ann. 1995, 241. (b) Catalán J., López V.; Pérez P. Liebigs Ann. 1995, 793. (c) Catalán, J.; Díaz, C.; López, V.; Pérez, P.; Paz, J.-L. de; Rodríguez ,J. G. Liebigs Ann. 1996, 1785. (d) Catalán J. Palomar J., Díaz C.; Paz J. L. de, J. Phys. Chem. A 1997, 101, 5183. (e) Catalán J. Díaz C. Liebigs Ann.1997, 1941. (f) Catalán J. Mena W., Meutermans W.; Elguero J. J. Phys Chem. 1992, 96, 3615. (g) Catalán J., Díaz C.; García-Blanco F. J. Org. Chem. 2000, 65, 9226. (h) idem íbid. 2001, 66, 5846.

7. Paéz, J. A.; Campillo, N.; Elguero, J. Gazzetta Chimica Italiana. 1996, 307.

8. (a) Altman D. G.; Bland J. M. The Statistician 1983, 32, 307. (b) Bland J. M.; Altman D. G. Lancet, 1986, 307. (c) ibid. 908. (d) Altman D. G.; Bland J. M. Applied Statistics 1987, 36, 224. (e) Bland J. M.; Altman D. G. Lancet 1995, 346:1085.

9. Exner, O. Correlation Analysis of Chemical Data, (Plenum Press, New York, and SNTL, Prague, 1988. 